\title{
A parametric study on the growth of yield front in rotating annular disks
}

\author{
Shubhankar Bhowmick ${ }^{1}$, Dipten Misra ${ }^{2}$ and Kashi Nath Saha ${ }^{3}$ \\ ${ }^{1}$ Department of Mechanical Engineering, National Institute of Technology, Raipur 492010, India \\ ${ }^{2,3}$ Department of Mechanical Engineering, Jadavpur University, Kolkata 700032, India \\ Email: kashinathsaha@gmail.com (telefax: +91 332414 6890)
}

\begin{abstract}
The growth of elastic-plastic front under post-elastic conditions in high speed rotating annular disks having exponential and parabolic geometry variation is investigated in the present study. The problem is addressed in post-elastic regime through an extension of variational method based on von-Mises yield criterion and linear strain hardening material behavior. Assuming a series expression of the unknown variable, the solution of the governing equation is obtained using Galerkin's principle. The approximate solution is obtained using an iterative method. The von-Mises stress distribution in the disk is reported in waterfall plots at various load steps corresponding to angular speed at the initiation of yielding till the attainment of fully plastic state. Effect of geometry parameters on the stress state of the disk is studied and the relevant results are reported in dimensionless form.
\end{abstract}

Keywords: Variational method, von-Mises stress, plastic front, limit angular speed, annular disk

\section{Introduction}

Due to its widespread applications in industry, the analysis of rotating disk has been of great interest to many researchers across the world. The study has become much more feasible in the past few decades due to the intensified application of numerical methods as well as advent of computational machines. It has been observed that thickness variation of disks results in higher operating speeds and reduced weight and rotary inertia as compared to those of uniform disks. Further, post-elastic analysis of rotating disks leads to efficient utilization the disk material. The present paper investigates the stress and deformation states of such annular disks with parabolic and exponential thickness variation.

The analysis of admissible angular speed of rotation for annular and solid disks of hyperbolically varying thickness, made of incompressible material, using large elastic deformation theory has been reported by Chaudhary and Gupta (1992). Güven (1992) investigated the effect of radial density gradient on elastic-plastic stresses and radial displacement of rotating annular disks with variable thickness and reported the influence of density gradient on angular velocity and elastic plastic interface radius. Eraslan and Argeso (2002) reported the elastic and plastic limit angular speeds for disks of variable thickness in power function. Based on von-Mises yield criterion and its flow rule the plastic limit angular speed is calculated for disks under linear as well as non-linear strain hardening behaviour. Results indicate increase in limit angular speed with reduction in thickness at the edge of the disk and reduction in disk mass due to shape of the profile. In another paper, analytical methods based on laws of equilibrium have been developed and reported by Eraslan and Apatay (2003) to study the elastic deformation and establish limit angular speeds of disks of parabolic thickness variation. The stresses and deformations of rotating constant and linearly varying thickness solid and annular disks under unified yield criterion were studied by Ma et al. (2001). In this criterion, with suitable adjustment of weighing coefficients one of Tresca's, von Mises or Yu criterion could be obtained. A semi analytical solution for an elastic-perfectly plastic annular rotating disk and its associated flow rule was presented and the effect of yield criterion on the size of the plastic zone was reported by Alexandrova and Alexandrov (2004). In another work, Alexendrova et al. (2004) developed a semi analytical method based on von Mises yield criterion and its associated flow rule to determine displacement field and strain distribution in a thin annular rotating disk of constant thickness. In a recent work Alexandrova and Villa Real (2007) reported the influences of rotational speed and thickness variation on the plastic solution of hyperbolic annular disks in terms of von-Mises yield criterion 
and its associated flow rule. Bhowmick et al. (2008) proposed a generalized variational method to study the behavior of externally loaded rotating solid disks of variable thickness in the elastic regime. Based on deformation theory of plasticity, the method has also been used into elasto-plastic domain under the assumption of von-Mises failure criterion with linear strain hardening theory (Bhowmick et al., 2006).

In the present study, a numerical method based on variational principle for elasto-plastic analysis of rotating annular disks having parabolic and exponential thickness variation has been proposed. A solution algorithm using von Mises yield criterion has been developed to obtain an approximate solution of the unknown displacement field. The governing set of equations is solved in an iterative manner and the validation of the proposed method with ANSYS is also presented. The coordinate functions used to approximate the radial displacement field are generated numerically following Gram-schimdt orthogonalization scheme. New results indicating the growth of the yield front in annular non-uniform disks have been presented for few different types of thickness variation.

\section{Mathematical formulation}

The rotational symmetry of the loading, geometry and boundary condition of a disk made of homogeneous and isotropic material makes the analysis axisymmetric. In the present study exponential and parabolic disk thickness variation of annular disks is considered. The disk subjected to rotational loading experiences radial and tangential strain. At a certain speed, known as elastic limit angular speed, the stress field of the disk attains the yield limit value at a certain location thus giving rise to a plastic front. The present method proposes a suitable iterative algorithm which can capture the location of plastic front numerically and take up the stress-strain analysis in post-elastic regime. Stress-strain relation of the disk is considered linear elastic followed by linear strain hardening (Eraslan and Argeso (2002) as shown in Figure 1. In the present paper, von Mises theory is taken as the failure criteria and under plane stress condition, the general condition of yielding is given by,

$$
\sqrt{\sigma_{r}^{2}-\sigma_{r} \sigma_{t}+\sigma_{t}^{2}}=\sigma_{y}
$$

where the left hand side of the equation gives von-Mises stress, $\sigma_{v m}$. The advantage in using von Mises criterion lies in the single equation based formulation that takes care of the whole plastic region. Whenever the von Mises stress at a particular radial location of a rotating disk reaches the uniaxial yield stress value, the plastic front initiates at that location and the corresponding rotational speed is termed as elastic limit angular speed $\left(\omega_{1}\right)$. On further increase in rotational speed, a certain region of the disk attains postelastic state and when this region encompasses the entire disk we get plastic limit speed or collapse speed $\left(\omega_{2}\right)$. It is important to note that the initiation of plastic front gives rise to non-homogeneity due to presence of separate regions with different flow characteristics depending on elasticity modulus in elastic region and tangent modulus in post-elastic region, however the assumption of isotropy remains valid for the entire disk. The limit elastic state followed by the advancement of the yield front till limit plastic state is reached is shown in Figure 2 (a-c). At elastic limit speed the von-Mises stress attains uniaxial yield stress value at the inner boundary of rotating annular disk (Figure 2 (a)) and as a consequence, the yield front starts propagating towards the outer edge with increase in rotational speed (Figure 2 (b)) until it attains a fully plastic state (Figure 2 (c)). The domain of the disk beyond elastic limit speed gets divided into two (inner plastic-outer elastic) regions, an inner plastic region ( $r=a$ to $r=r_{y}$ ) and an outer elastic region $\left(r=r_{y}\right.$ to $r=b$ ). However for certain thickness variation based on the selected geometry parameters and loading conditions, an annular disk may also start yielding at an intermediate radial location but such cases has not been considered for the present study.

The variational form of total potential energy states that the extremum of the summation of strain energy $(U)$ and work potential $(V)$ is conserved for a system in equilibrium, i.e.,

$$
\delta(U+V)=0
$$

The total strain energy $U$ consists of outer elastic $\left(U_{e}\right)$ and inner plastic $\left(U_{p}\right)$ part and $V$ is the potential of the external forces. The strain energy in the disk is obtained by evaluating the integral $\frac{1}{2} \int\left(\sigma_{i j} \varepsilon_{i j}\right) d v$ and for a plane stress condition this is equivalent to computing $\frac{1}{2} \int_{V o l}\left(\sigma_{t} \varepsilon_{t}+\sigma_{r} \varepsilon_{r}\right) d v$.

Expression of the elastic part of strain energy for the outer region (for $r=r_{y}$ to $r=b$ ) is given by (Bhowmick et al., 2008),

$$
U_{e}=\frac{\pi E}{1-v^{2}} \int_{r_{y}}^{b}\left\{\frac{u^{2}}{r}+2 v u\left(\frac{d u}{d \mathrm{r}}\right)+r\left(\frac{d u}{d r}\right)^{2}\right\} h d r .
$$




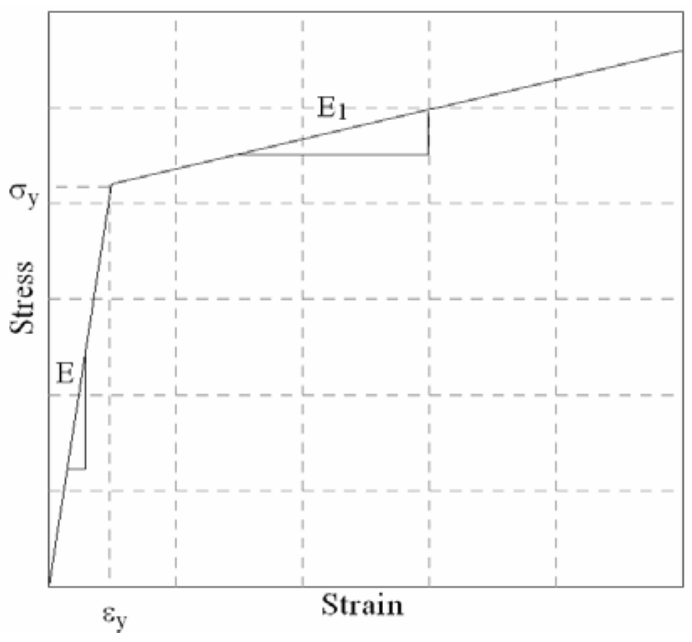

Figure 1. Linear strain hardening material behavior.

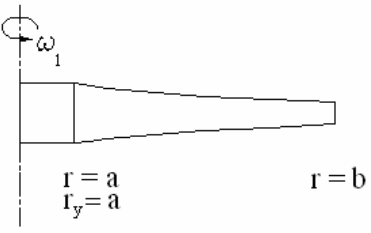

(a)

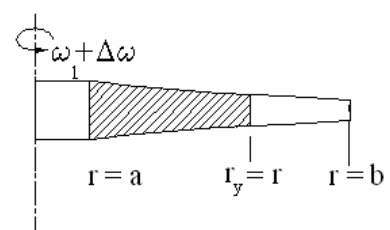

(b)

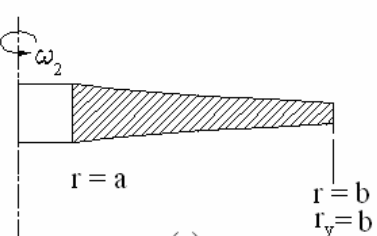

(c)

Figure 2 Propagation of elastic-plastic interface with increasing angular speed in annular disks:

(a) initiation at root (b) at intermediate location and (c) collapse at the outer radius

In arriving at these equations, the following strain-displacement and stress-strain relations have been used,

and

$$
\varepsilon_{r}=d u / d r, \varepsilon_{t}=u / r
$$

$$
\sigma_{r}=\frac{E}{\left(1-v^{2}\right)}\left[\varepsilon_{r}+v \varepsilon_{t}\right], \sigma_{t}=\frac{E}{\left(1-v^{2}\right)}\left[\varepsilon_{t}+v \varepsilon_{r}\right]
$$

Here $u$ is the displacement field in radial direction. Post-elastic part of strain energy, $U_{p}$ (for $r=a$ to $r=r_{y}$ ), is obtained from the integral $\int_{v o l}($ area under radial ' $\sigma-\varepsilon$ ' curve + area under tangential ' $\sigma-\varepsilon$ ' curve $) d v$, i.e, $U_{p}=\int_{v o l}\left(d U_{r}+d U_{t}\right) d v$, where, $U_{r}$ and $U_{t}$ are the contributions coming from radial and tangential stresses and strains. To develop the mathematical expression of these energies, we note that the strain-displacement and stress-strain relations in the post-elastic region becomes,

$$
\frac{d u}{d r}=\varepsilon_{r}^{p}+\varepsilon_{r}^{0}, \frac{u}{r}=\varepsilon_{t}^{p}+\varepsilon_{t}^{0}
$$

and using deformation theory of plasticity and linear strain hardening behaviour the following relations are obtained,

$$
\begin{aligned}
& \sigma_{r}^{0}=\frac{E}{\left(1-v^{2}\right)}\left[\varepsilon_{r}^{0}+v \varepsilon_{t}^{0}\right], \sigma_{t}^{0}=\frac{E}{\left(1-v^{2}\right)^{L}}\left[\varepsilon_{t}^{0}+v \varepsilon_{r}^{0}\right] . \\
& \sigma_{r}^{p}=\frac{E_{1}}{\left(1-v_{p}^{2}\right)}\left[\varepsilon_{r}^{p}+v_{p} \varepsilon_{t}^{p}\right], \sigma_{t}^{p}=\frac{E_{1}}{\left(1-v_{p}{ }^{2}\right)}\left[\varepsilon_{t}^{p}+v_{p} \varepsilon_{r}{ }^{p}\right] .
\end{aligned}
$$

Here, $\varepsilon_{r}^{0}, \varepsilon_{t}^{0}$ and $\sigma_{r}^{0}, \sigma_{t}^{0}$ are the strains and stresses in radial and tangential direction in yield condition respectively. With proper substitutions the following relations for $d U_{r}$ and $d U_{t}$ are obtained. 


$$
\begin{aligned}
d U_{r} & =\frac{1}{2} \frac{E_{1}}{\left(1-v_{p}{ }^{2}\right)}\left[\left(\frac{d u}{d r}\right)^{2}+v_{p} \frac{d u}{d r} \frac{u}{r}\right]+\frac{E}{\left(1-v^{2}\right)}\left[\varepsilon_{r}{ }^{0}+v \varepsilon_{t}{ }^{0}\right] \frac{d u}{d r}-\frac{E_{1}}{\left(1-v_{p}^{2}\right)}\left[\varepsilon_{r}{ }^{0}+v_{p} \varepsilon_{t}{ }^{0}\right] \frac{d u}{d r} \\
& +\frac{1}{2} \frac{E_{1}}{\left(1-v_{p}{ }^{2}\right)}\left[\left(\varepsilon_{r}{ }^{0}\right)^{2}+v_{p} \varepsilon_{r}{ }^{0} \varepsilon_{t}^{0}\right]-\frac{1}{2} \frac{E}{\left(1-v^{2}\right)}\left[\left(\varepsilon_{r}^{0}\right)^{2}+v \varepsilon_{r}^{0} \varepsilon_{t}^{0}\right]+\frac{1}{2} \frac{E_{1}}{\left(1-v_{p}{ }^{2}\right)} v_{p}\left[\frac{d u}{d r} \varepsilon_{t}^{0}-\frac{u}{r} \varepsilon_{r}^{0}\right]
\end{aligned}
$$

and

$$
\begin{aligned}
d U_{t} & =\frac{1}{2} \frac{E_{1}}{\left(1-v_{p}^{2}\right)}\left[\left(\frac{u}{r}\right)^{2}+v_{p} \frac{d u}{d r} \frac{u}{r}\right]+\frac{E}{\left(1-v^{2}\right)}\left[\varepsilon_{t}^{0}+v \varepsilon_{r}{ }^{0}\right] \frac{u}{r}-\frac{E_{1}}{\left(1-v_{p}{ }^{2}\right)}\left[\varepsilon_{t}^{0}+v_{p} \varepsilon_{r}{ }^{0}\right] \frac{u}{r} \\
& +\frac{1}{2} \frac{E_{1}}{\left(1-v_{p}{ }^{2}\right)}\left[\left(\varepsilon_{t}^{0}\right)^{2}+v_{p} \varepsilon_{r}{ }^{0} \varepsilon_{t}^{0}\right]-\frac{1}{2} \frac{E}{\left(1-v^{2}\right)}\left[\left(\varepsilon_{t}^{0}\right)^{2}+v \varepsilon_{r}{ }^{0} \varepsilon_{t}^{0}\right]+\frac{1}{2} \frac{E_{1}}{\left(1-v_{p}{ }^{2}\right)} v_{p}\left[\frac{u}{r} \varepsilon_{r}^{0}-\frac{d u}{d r} \varepsilon_{t}^{0}\right] .
\end{aligned}
$$

Thus the strain energy in the inner post-elastic region is obtained from the expression

$$
\begin{aligned}
U_{p} & =\frac{1}{2} \frac{E_{1}}{\left(1-v_{p}^{2}\right)} \int_{a}^{r_{y}}\left\{\frac{u^{2}}{r}+r\left(\frac{d u}{d r}\right)^{2}+2 v_{p} u \frac{d u}{d r}\right\} 2 \pi h d r+\frac{E}{\left(1-v^{2}\right)} \int_{a}^{r_{y}}\left\{\left(\varepsilon_{r}^{0}+v \varepsilon_{t}^{0}\right) r \frac{d u}{d r}+\left(\varepsilon_{t}^{0}+v \varepsilon_{r}^{0}\right) u\right\} 2 \pi h d r \\
& -\frac{E_{1}}{\left(1-v_{p}^{2}\right)} \int_{a}^{r_{y}}\left\{\left(\varepsilon_{r}^{0}+v_{p} \varepsilon_{t}^{0}\right) r \frac{d u}{d r}+\left(\varepsilon_{t}^{0}+v_{p} \varepsilon_{r}^{0}\right) u\right\} 2 \pi h d r-\frac{E}{2\left(1-v^{2}\right)} \int_{a}^{r_{y}}\left\{\left(\varepsilon_{r}^{0}\right)^{2} r+\left(\varepsilon_{t}^{0}\right)^{2} r+2 v \varepsilon_{t}^{0} \varepsilon_{r}^{0} r\right\} 2 \pi h d r \\
& +\frac{E_{1}}{2\left(1-v_{p}^{2}\right)} \int_{a}^{r_{y}}\left\{\left(\varepsilon_{r}^{0}\right)^{2} r+\left(\varepsilon_{t}^{0}\right)^{2} r+2 v_{p} \varepsilon_{t}^{0} \varepsilon_{r}^{0} r\right\} 2 \pi h d r .
\end{aligned}
$$

The expression for work potential is as follows

$$
V=-2 \pi \rho \omega^{2} \int_{a}^{b}\left(r^{2} u h\right) d r
$$

In the mathematical expressions, the normalization of the energy expressions is carried out with three parameters $\Delta, \Delta_{1}$ and $\Delta_{2}$

$$
\Delta=b-a, \Delta_{1}=r_{y}-a \text { and } \Delta_{2}=b-r_{y} .
$$

Accordingly three normalized coordinates $\xi$, $\xi_{1}$ and $\xi_{2}$ are obtained as shown below

$$
\xi=(r-a) / \Delta, \xi_{1}=(r-a) / \Delta_{1} \text { and } \xi_{2}=\left(r-r_{y}\right) / \Delta_{2}
$$

Substituting the normalized expressions of the energies (Eq. 3, 4 and 5) in Eq. (2), the governing equilibrium equation becomes,

$$
\begin{aligned}
& \frac{E}{1-v^{2}} \int_{0}^{1}\left\{\frac{u \delta u}{\left(\Delta_{2} \xi_{2}+r_{y}\right)}+\frac{v}{\Delta_{2}}\left[u \delta\left(\frac{d u}{d \xi_{2}}\right)+\frac{d u}{d \xi_{2}} \delta u\right]+\frac{\left(\Delta_{2} \xi_{2}+r_{y}\right)}{\left(\Delta_{2}\right)^{2}} \frac{d u}{d \xi_{2}} \delta\left(\frac{d u}{d \xi_{2}}\right)\right\} h \Delta_{2} d \xi_{2} \\
& +\frac{E_{1}}{\left(1-v_{p}^{2}\right)} \int_{0}^{1}\left[\left\{\frac{\left(\Delta_{1} \xi_{1}\right)}{\left(\Delta_{1}\right)^{2}} \frac{d u}{d \xi_{1}} \delta\left(\frac{d u}{d \xi_{1}}\right)+\frac{u \delta u}{\left(\Delta_{1} \xi_{1}\right)}\right\}+\frac{v_{p}}{\Delta_{1}}\left\{\frac{d u}{d \xi_{1}} \delta u+u \delta\left(\frac{d u}{d \xi_{1}}\right)\right\} h \Delta_{1} d \xi_{1}\right. \\
& +\frac{E}{\left(1-v^{2}\right)} \int_{0}^{1}\left\{\varepsilon_{r}^{0}\left(v \delta u+\frac{\left(\Delta_{1} \xi_{1}\right)}{\Delta_{1}} \delta\left(\frac{d u}{d \xi_{1}}\right)\right)+\varepsilon_{t}^{0}\left(\delta u+v \frac{\left(\Delta_{1} \xi_{1}\right)}{\Delta_{1}} \delta\left(\frac{d u}{d \xi_{1}}\right)\right)\right\} h \Delta_{1} d \xi_{1} \\
& -\frac{E_{1}}{\left(1-v_{p}^{2}\right)} \int_{0}^{1}\left\{\varepsilon_{r}^{0}\left(v_{p} \delta u+\frac{\left(\Delta_{1} \xi_{1}\right)}{\Delta_{1}} \delta\left(\frac{d u}{d \xi_{1}}\right)\right)+\varepsilon_{t}^{0}\left(\delta u+v_{p} \frac{\left(\Delta_{1} \xi_{1}\right)}{\Delta_{1}} \delta\left(\frac{d u}{d \xi_{1}}\right)\right)\right\} h \Delta_{1} d \xi_{1} \\
& -\rho \omega^{2} \int_{0}^{1}\left\{(\Delta \xi)^{2} h \delta u\right\} \Delta d \xi=0
\end{aligned}
$$


The global displacement field $u(\xi)$ in the above equation is approximated by $u(\xi) \cong \sum c_{i} \phi_{i}$, where $\phi_{i}$ is the set of orthogonal functions developed by satisfying the relevant boundary conditions $\left(\left.\sigma_{r}\right|_{(a)}=0\right.$ and $\left.\left.\sigma_{r}\right|_{(b)}=0\right)$ of an annular rotating disk in elastic regime. For the purpose of computation, displacement functions in the post-elastic and elastic regions are expressed as $u\left(\xi_{1}\right) \cong \sum c_{i} \phi_{i}{ }^{p}$ and $u\left(\xi_{2}\right) \cong \sum c_{i} \phi_{i}{ }^{e}$ respectively, where $\phi_{i}^{p}$ and $\phi_{i}^{e}$ are portions of the global function $\phi_{i}$ in post-elastic and elastic region. Substituting these assumed displacement functions and replacing operator $\delta$ by $\partial / \partial c_{j}$ the governing equation is obtained in matrix form as follows:

$$
\begin{aligned}
& \frac{E}{1-v^{2}} \sum_{j=1}^{n f} \sum_{i=1}^{n f} c_{i} \int_{0}^{1}\left\{\frac{\phi_{i}^{e} \phi_{j}^{e}}{\left(\Delta_{2} \xi_{2}+r_{c}\right)}+\frac{v}{\Delta_{2}}\left(\phi_{i}^{e^{\prime}} \phi_{j}^{e}+\phi_{i}^{e} \phi_{j}^{e^{\prime}}\right)+\frac{\left(\Delta_{2} \xi_{2}+r_{c}\right)}{\left(\Delta_{2}\right)^{2}} \phi_{i}^{e^{\prime}} \phi_{j}^{e^{\prime}}\right\} h \Delta_{2} d \xi_{2} \\
& +\frac{E_{1}}{\left(1-v_{p}^{2}\right)} \sum_{j=1}^{n f} \sum_{i=1}^{n f} c_{i} \int_{0}^{1}\left[\left\{\frac{\left(\Delta_{1} \xi_{1}\right)}{\left(\Delta_{1}\right)^{2}} \phi_{i}^{p^{\prime}} \phi_{j}^{p^{\prime}}\right\}+\left\{\frac{\phi_{i}^{p} \phi_{j}^{p}}{\left(\Delta_{1} \xi_{1}\right)}\right\}+\frac{v_{p}}{\Delta_{1}}\left\{\phi_{i}^{p^{\prime}} \phi_{j}^{p}+\phi_{i}^{p} \phi_{j}^{p^{\prime}}\right\}\right] h \Delta_{1} d \xi_{1} \\
& =\rho \omega^{2} \sum_{j=1}^{n f} \int_{0}^{1}\left\{(\Delta \xi)^{2} \phi_{j}\right\} h \Delta d \xi \\
& -\frac{E}{\left(1-v^{2}\right)} \sum_{j=1}^{n f} \int_{0}^{1}\left[\varepsilon_{r}^{0}\left\{v \phi_{j}^{p}+\frac{\left(\Delta_{1} \xi_{1}\right)}{\Delta_{1}} \phi_{j}^{p^{\prime}}\right\}+\varepsilon_{t}^{0}\left\{\phi_{j}^{p}+v \frac{\left(\Delta_{1} \xi_{1}\right)}{\Delta_{1}} \phi_{j}^{p^{\prime}}\right\}\right] h \Delta_{1} d \xi_{1} \\
& +\frac{E_{1}}{\left(1-v_{p}^{2}\right)} \sum_{j=1}^{n f} \int_{0}^{1}\left[\varepsilon_{r}^{0}\left\{v_{p} \phi_{j}^{p}+\frac{\left(\Delta_{1} \xi_{1}\right)}{\Delta_{1}} \phi_{j}^{p^{\prime}}\right\}+\varepsilon_{t}^{0}\left\{\phi_{j}^{p}+v_{p} \frac{\left(\Delta_{1} \xi_{1}\right)}{\Delta_{1}} \phi_{j}^{p^{\prime}}\right\}\right] h \Delta_{1} d \xi_{1}
\end{aligned}
$$

In this equation ( $)^{\prime}$ indicates differentiation with respect to normalized coordinates.

2.1 Solution algorithm: The governing equation can be expressed in matrix form as, $[K]\{c\}=\{f\}$ and the required solution of unknown coefficients $\{c\}$ is obtained numerically by using an iterative scheme involving two loops. The rotational speed is incremented through a suitable step size $\left(\Delta \Omega_{1}\right)$ in the (first) outer loop and interface radius $r_{y}$ is given a small increment in the inner (second) loop. For a particular load step above elastic limit angular speed, the values of $\varepsilon_{r}^{0}$ and $\varepsilon_{t}^{0}$ are necessary to generate the right hand side of Eq.(7). To this end, it is assumed that the ratio of tangential and radial stress when yielding occurs at each point in the plastic region in the present load step is same as the ratio of tangential and radial stress obtained in the preceding solved load step. For each load step, the location of plastic front is given a small increment starting from its previous location. From this defined limits of elastic and plastic zones the solution of the displacement field is obtained and the stress values are computed at the assumed elasto-plastic interface. The value of von Mises stress at the assumed plastic front location is checked for the present load step and equality of the value with that of yield stress gives the required solution for that load step. All relevant data are stored at this instant and it is followed by a load increment by returning to outer loop till the disk attains a fully plastic state. A brief description of the solution algorithm which presupposes solution of limit angular speed $\Omega_{1}$ and yield front location $r_{y}$ is provided below.

1. Two loops are commenced with $r_{y}$ initiated to the value of $a$.

2. The rotational speed is incremented through a suitable step size $\left(\Delta \Omega_{1}\right)$ in the outer (first) loop.

3. Inner (second) loop is initiated and $r_{y}$ is given a small increment.

4. The ratio of $\sigma_{r}^{0}$ and $\sigma_{t}^{0}$ in the elastic region is constant for a particular radial location. That ratio ' $\lambda$ ' is stored during elastic analysis. Whenever yield front reaches a particular radial location, its von-Mises stress reaches the yield stress. Corresponding to this value of von-Mises stress, the values of $\sigma_{r}^{0}$ and $\sigma_{t}^{0}$ at any point in the plastic region can be obtained from the relation

$$
\begin{aligned}
& \sigma_{v m}=\sigma_{t}\left(1-\lambda+\lambda^{2}\right)^{\frac{1}{2}}, \\
& \text { where } \frac{\sigma_{r}}{\sigma_{t}}=\lambda
\end{aligned}
$$

5. The displacement field is then obtained using the elastic-plastic formulation of Eq. (7).

6. A check is performed on von Mises stress and if it attains the value of yield stress at the yield front location then current position is the exact yield front location. This ensures solution for the current load step, when the relevant results are 
noted and followed by a load increment by returning to outer loop mentioned in step 2. Otherwise the program returns to step 3 so long the plastic region covers whole of the disk.

2.2. Determination of plastic strain: At $\Omega>\Omega_{1}$, radial and tangential strains at each coordinate inside the post-elastic region of the disk can be decomposed into elastic and plastic parts as given below.

$$
\begin{aligned}
& \varepsilon_{r}=\varepsilon_{r}{ }^{e}+\varepsilon_{r}{ }^{p} \\
& \varepsilon_{t}=\varepsilon_{t}{ }^{e}+\varepsilon_{t}{ }^{p}
\end{aligned}
$$

where the superscript ' $e$ ' and ' $p$ ' denote the elastic and plastic part of the strains respectively.

The relations between stresses and elastic strains can be derived from generalized Hooke's law. For a state of plane stress $\left(\sigma_{z}=0\right.$ ), assuming Hencky's deformation theory of plasticity, the plastic strain components are given as

$$
\begin{gathered}
\varepsilon_{r}^{p}=\frac{\varepsilon_{v m}^{p}}{\sigma_{v m}}\left(\sigma_{r}-\frac{\sigma_{t}}{2}\right) \\
\varepsilon_{t}^{p}=\frac{\varepsilon_{v m}^{p}}{\sigma_{v m}}\left(\sigma_{t}-\frac{\sigma_{r}}{2}\right)
\end{gathered}
$$

In Eq. (10a, b) $\varepsilon_{v m}^{p}$ is the equivalent plastic strain and $\sigma_{v m}$ is the equivalent or von-Mises stress. Based on linear strain hardening material behavior of the disk material, the relation between equivalent plastic strain and von-Mises stress can be expressed as

$$
\varepsilon_{v m}^{p}=\left(\frac{\sigma_{v m}-\sigma_{y}}{H}\right)
$$

where $H$ is the hardening parameter and is given by

$$
H=\frac{E E_{1}}{E-E_{1}}
$$

\section{Results and discussions}

The results are presented in terms of the following dimensionless and normalized variables: angular velocity $\Omega=\omega b \sqrt{\rho / \sigma_{y}}$, stress $\bar{\sigma}=\sigma / \sigma_{y}$, displacement $\bar{u}=u E / b \sigma_{y}$ and hardening parameter $\bar{H}=E_{1} /\left(E-E_{1}\right)$. Results for post-elastic behaviour of linear strain hardening material have been generated using the values $\bar{H}=0.5, a=0.2, b=1.0 \mathrm{~m}, \rho=7850 \mathrm{~kg} / \mathrm{m}^{3}, E=210 \mathrm{GPa}$ and $\sigma_{y}=350 \mathrm{MPa}$. The value of Poisson's ratio $v$ is taken as $1 / 3$ and $v_{p}$ is taken as 0.5 . The variation in disk geometry is controlled through parameters $n$ and $k$. The disk thickness variation is modeled using parabolic (type D1) and exponential (type D2) relations respectively as defined below:

$$
\begin{array}{ll}
h(\xi)=h_{0}\left[1-n \xi^{k}\right] & \text { for parabolic variation, } \\
h(\xi)=h_{0} \exp \left[-n \xi^{k}\right] & \text { for exponential disk, }
\end{array}
$$

where $h_{0}$ is the disk thickness at the root $(r=a)$

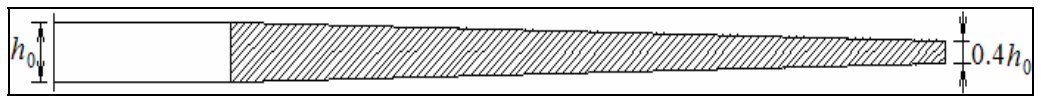

Figure 3 Tapered annular disk geometry $(n=0.6, k=1.0)$ used for validation 
A convergence study on the number of functions on limit elastic $\left(\Omega_{1}\right)$ and fully plastic $\left(\Omega_{2}\right)$ angular speed is carried out for an annular disk of uniform thickness with geometry parameters, $n=0.0$ and is reported in Table 1 . Based on these studies it is observed that good convergence is achieved with 8 coordinate functions and hence subsequent calculations are carried out at these many functions. All the functions are denoted by 24 Gauss points and an increment of 0.0001 radian per second is considered for increase in angular speed.

Table 1.Convergence study on number of co-ordinate functions

\begin{tabular}{|c|c|c|c|}
\hline S.No. & $n f$ & $\Omega_{1}$ & $\Omega_{2}$ \\
\hline 1 & 4 & 1.9064 & 1.9112 \\
\hline 2 & 5 & 1.0964 & 1.9086 \\
\hline 3 & 6 & 1.0964 & 1.9109 \\
\hline 4 & 7 & 1.0964 & 1.9110 \\
\hline 5 & 8 & 1.0965 & 1.9113 \\
\hline 6 & 9 & 1.0965 & 1.9113 \\
\hline 7 & 10 & 1.0965 & 1.9113 \\
\hline
\end{tabular}

For validation of the mathematical formulation a linearly tapered disk (refer Figure 3 ) is considered and comparison of result is carried out with ANSYS v11.0. The taper disk profile is obtained by considering geometry parameters $n=0.6$ and $k=1.0$ in Eq. 13. In ANSYS the disk of given geometry and identical material properties is modeled as an axisymmetric trapezoidal area and is meshed with plane-82 (solid) elements with a mapped mesh density of 6 by 50 . The model is centrifugally loaded by applying global angular velocity about the axis of rotation. Firstly the propagation of yield front with increment in angular speed is validated and is plotted in dimensionless form in Figure 4. The normalized displacement and stress values are validated at different load steps next and are plotted in Figure 5a-e. The first load step selected for validation corresponds to elastic limit angular speed i.e. at $\Omega_{1}=1.2414$ (Figure 5 a), and the speeds at which it reaches $\xi=0.25,0.50$ and 0.75 are the subsequent load steps chosen for validation. Hence the validation (Figure $5 \mathrm{~b}-\mathrm{d}$ ) is carried out for $\Omega$ values of 1.6605, 1.8169 and 1.9731 respectively. The final load step for validation is selected corresponding to $\Omega_{2}=2.1175$ at which the disk attains fully plastic state. These plots exhibit good agreement establishing validity of the present elasto-plastic analysis method.

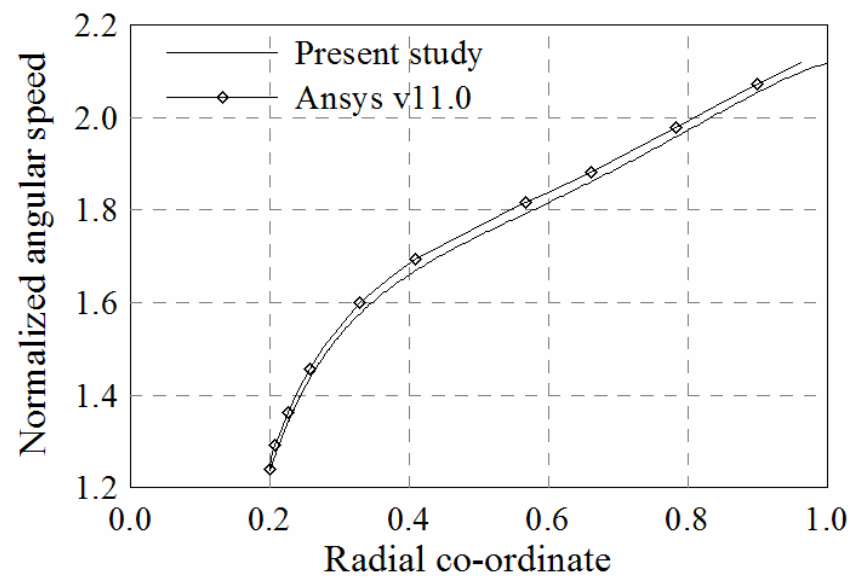

Figure 4 Validation study on propagation of yield front of a taper disk

The effect of hardening parameter is studied next. The geometry of disk selected is identical to the one used for validation. The comparison of yield front propagation with increasing angular speed for four different values of $\bar{H}(0.00,0.25,0.50$ and 0.75$)$ is plotted in dimensionless form in Figure 6 followed by a comparative plot of normalized displacement and von-Mises stress at fully plastic state in Figure 7 a-b.

Next a parametric study of annular disk in elasto-plastic regime for five different profiles each of parabolic (D1) and exponential (D2) geometries are considered. The disk profiles are shown in Figure 8. The yield front propagation of parabolic (D1) disks is plotted in dimensionless form in Figure 9 a-e followed by waterfall plots of normalized von-Mises stress in Figure 10 a-e. In these figures, dimensionless speed is incremented from $\Omega_{1}$ to $\Omega_{2}$ at equal intervals of step size 0.0025 . It is evident from Figure 9 a-e that for parabolic disks with increase in $\boldsymbol{n}$ the elastic and fully plastic speed increases where as for a given value of $\boldsymbol{n}$, if $\boldsymbol{k}$ is increased the limit speed tend to reduce. It is also indicative of the fact that with increase in $\boldsymbol{n}$ operating range increases while at a given value of $\boldsymbol{n}$, increase in $\boldsymbol{k}$ reduces the operating range for parabolic disks. Operating range is considered as the difference 
between fully plastic speed and limit elastic speed The plots of radial and tangential plastic strain at fully plastic speed for the respective disk geometries of type D1 is shown in Figure 11 a-b.
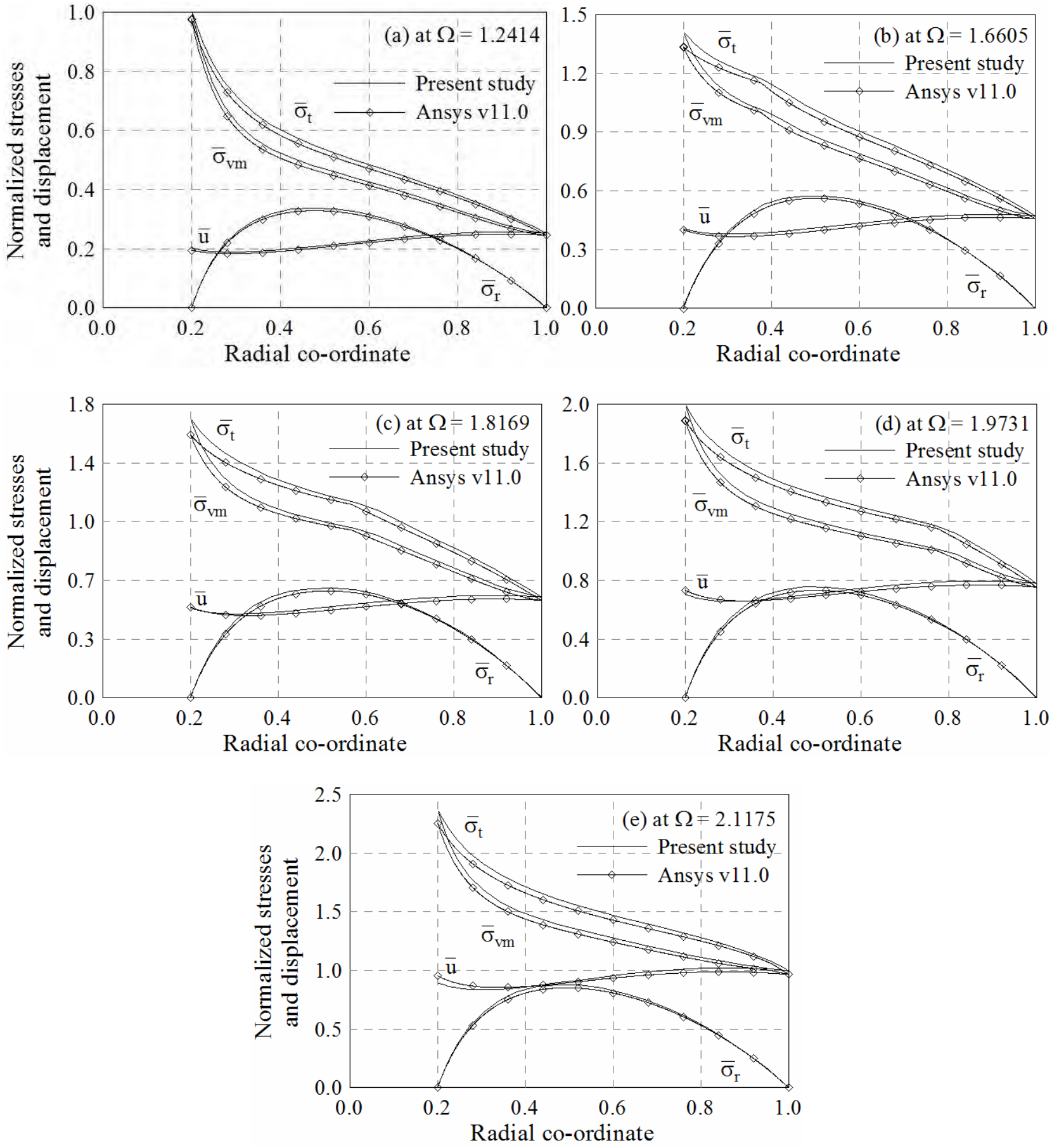

Figure 5a-e Normalized displacement and stress values at different angular speeds and their validation 


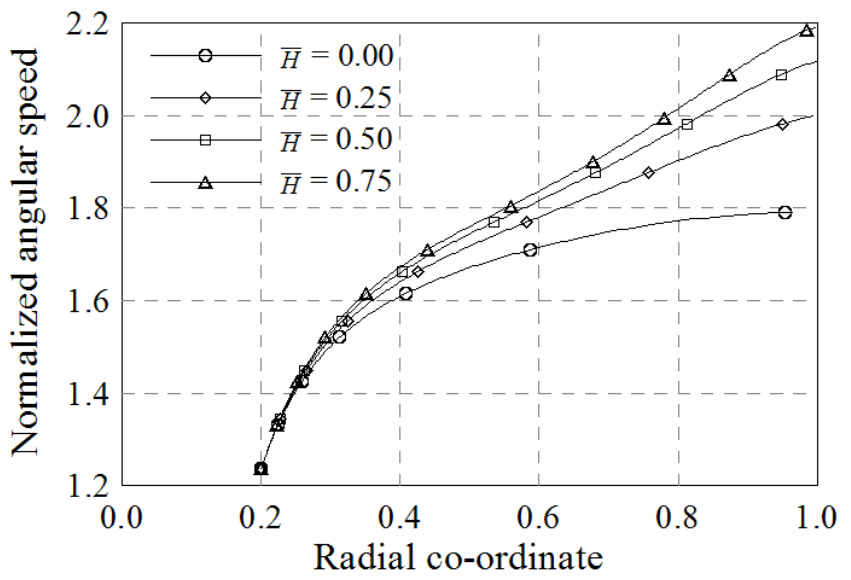

Figure 6 Comparative study of yield front propagation with angular speed for different hardening parameters
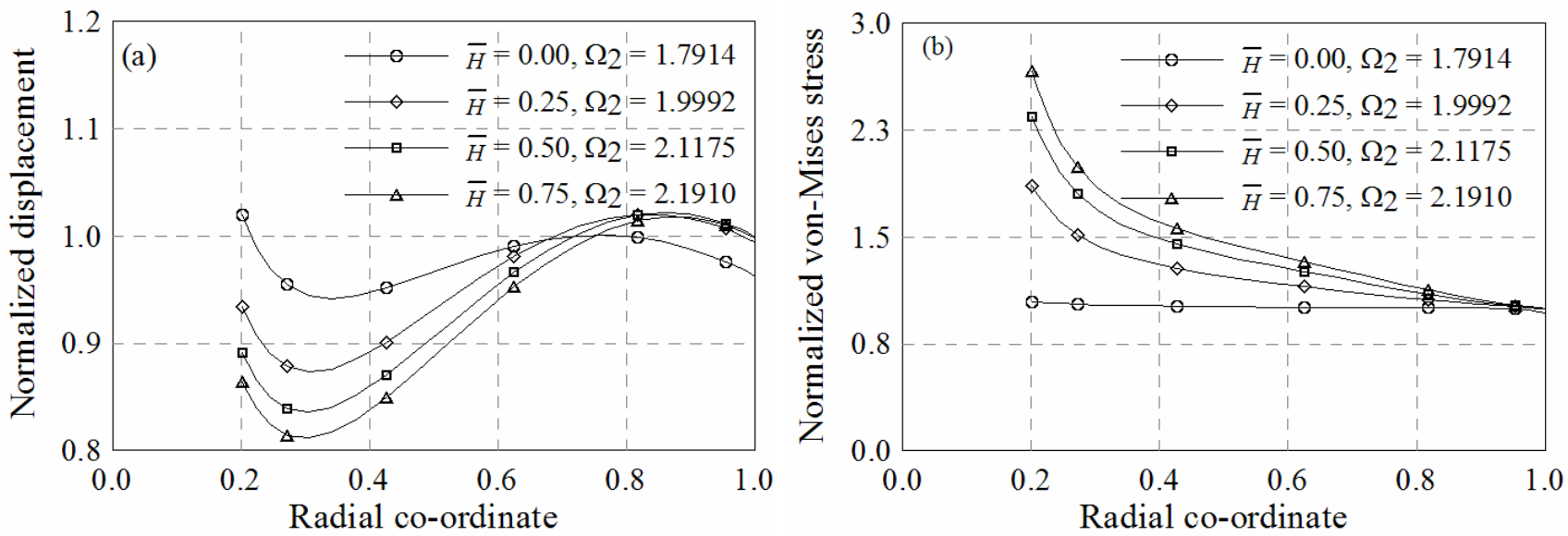

Figure 7a-b Comparative plots of normalized displacement and von-Mises stress for different hardening parameters

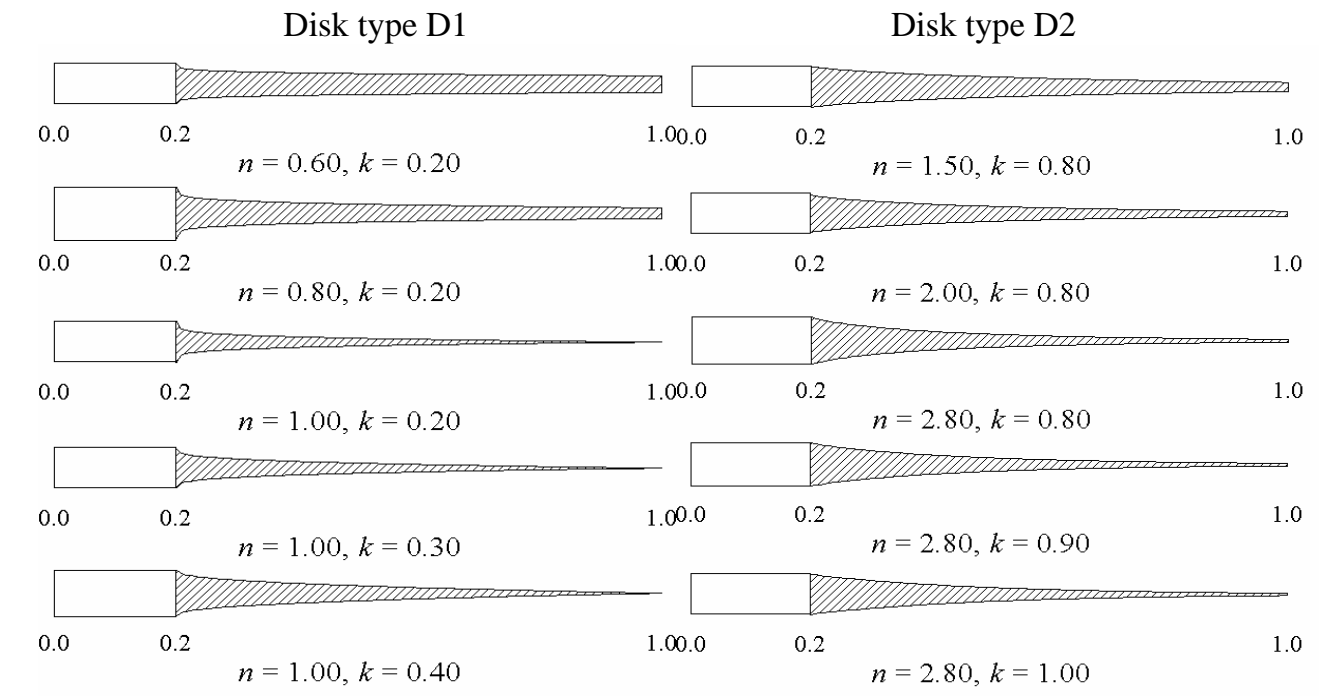

Figure 8 Various thickness profiles with corresponding geometry parameters for annular disks

Similar plots of yield front propagation and waterfall plots of von-Mises stress are plotted in Figure 12 a-e and Figure 13 a-e for exponential (D2) disk geometry. It is observed that for exponential disks increase in $\boldsymbol{n}$ as well as $\boldsymbol{k}$ increases both limit elastic speed and fully plastic speed. Furthermore it is also observed that the operating range of disks in post-elastic state increases with increase in $\boldsymbol{n}$ as well as $\boldsymbol{k}$. In Figure $14 \mathrm{a}$-b, the radial and tangential plastic strains for different disk geometries of disk type D2 are 
plotted. In general, the difference of fully plastic and limit elastic speed can be used as an index to measure the extent of effective utilization of disk material due to its thickness variation. On this basis it is observed that among the selected disk profiles the most effective ones are of geometry parameters $\boldsymbol{n}=1.0$ and $\boldsymbol{k}=0.2$ for disk type D1 and of $\boldsymbol{n}=2.8$ and $\boldsymbol{k}=1.0$ for disk type D2.
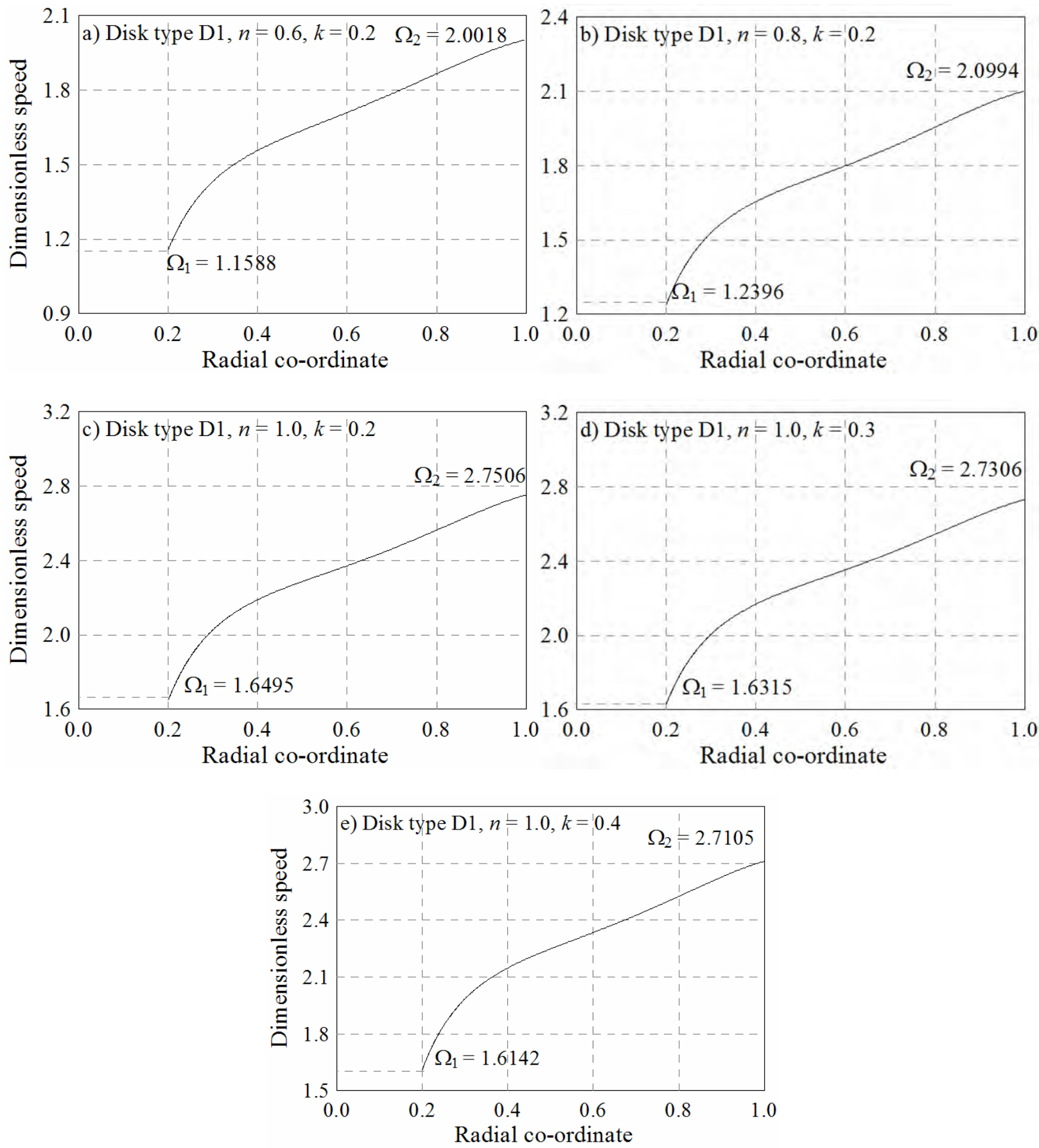

Figure 9a-e Plot of propagation of yield front with increase in angular speed for various parabolic annular disk geometries 

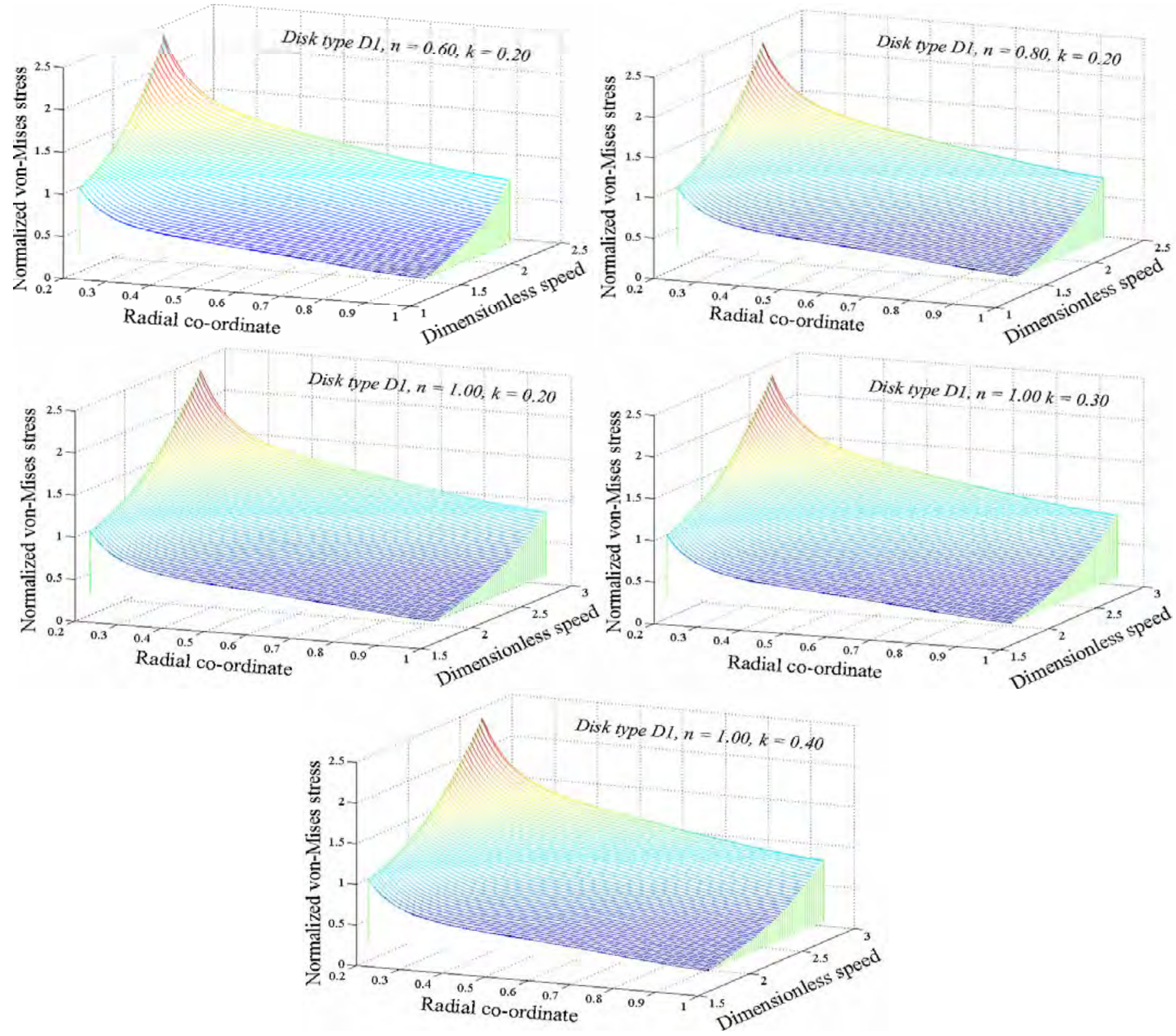

Figure 10 a-e Waterfall plots of variation of von-Mises stress with angular speed for various parabolic annular disks
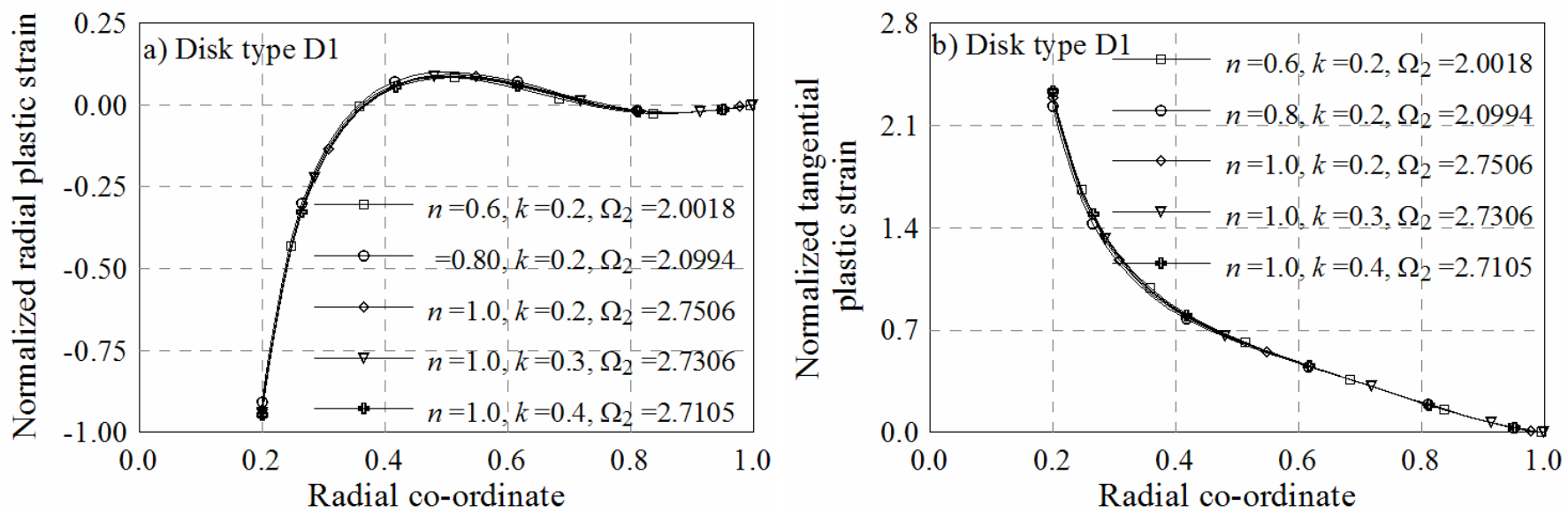

Figure 11a-b Plots of radial and tangential plastic strain for different disk geometries of disk type D1 

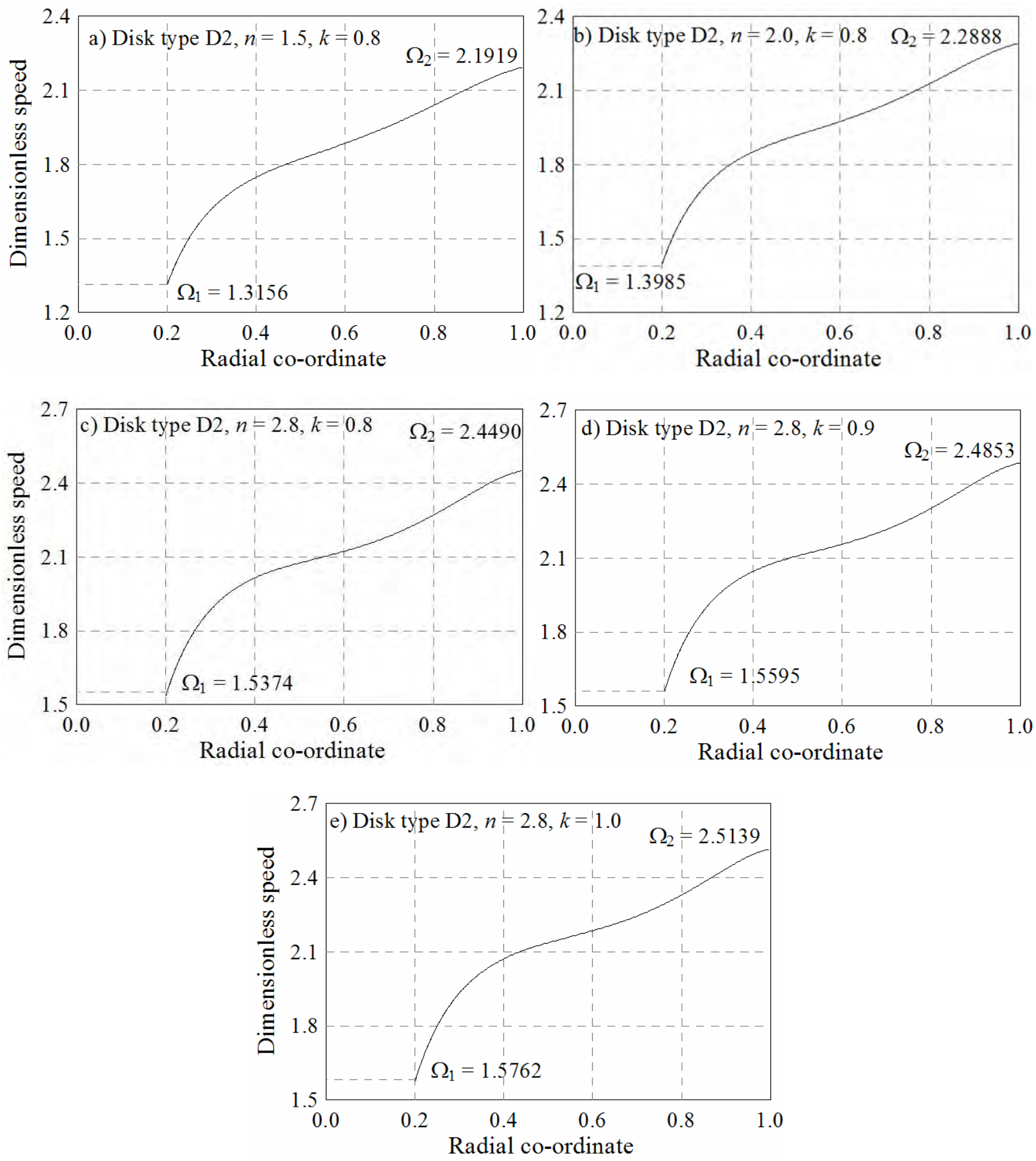

Figure 12a-e Plot of propagation of yield front with increase in angular speed for various exponential annular disk geometries

\section{Conclusion}

The post-elastic investigation of high-speed variable geometry rotating disks is formulated through a variational method assuming linear strain hardening material behavior following von Mises yield criterion and solution is obtained by assuming a series solution of the unknown displacement field. The results obtained by the present methodology have been validated and it showed a close conformity with the existing results of similar problem. The present formulation provides an easy user interface to carry out the parametric study of high speed rotating disks. It is a method based on full-domain study and unlike FEM it does not break the domain into finite elements and hence does not require the extensive and repeated pre-processing, solution and postprocessing computations. Hence the present method saves computational effort and time as compared to commercial Finite Element codes. Some new results for displacement field and radial, tangential and von-Mises stress field at plastic limit speed for parabolic and exponential disks for linear strain hardening material behavior have been furnished and plots showing the 
advancement of the plastic front with increase in rotational speed have been presented. The limit load factor, obtained from the study would be of much help to the design and application engineers. The method of formulation readily gives the kernel for dynamic analysis and many other complicating effects. The results are presented graphically so that they become designer friendly. The method developed has application potential in various other problems, e.g., shrink fitted rotating disk, pre-stressed rotating disk, compound disk made from different materials, etc.
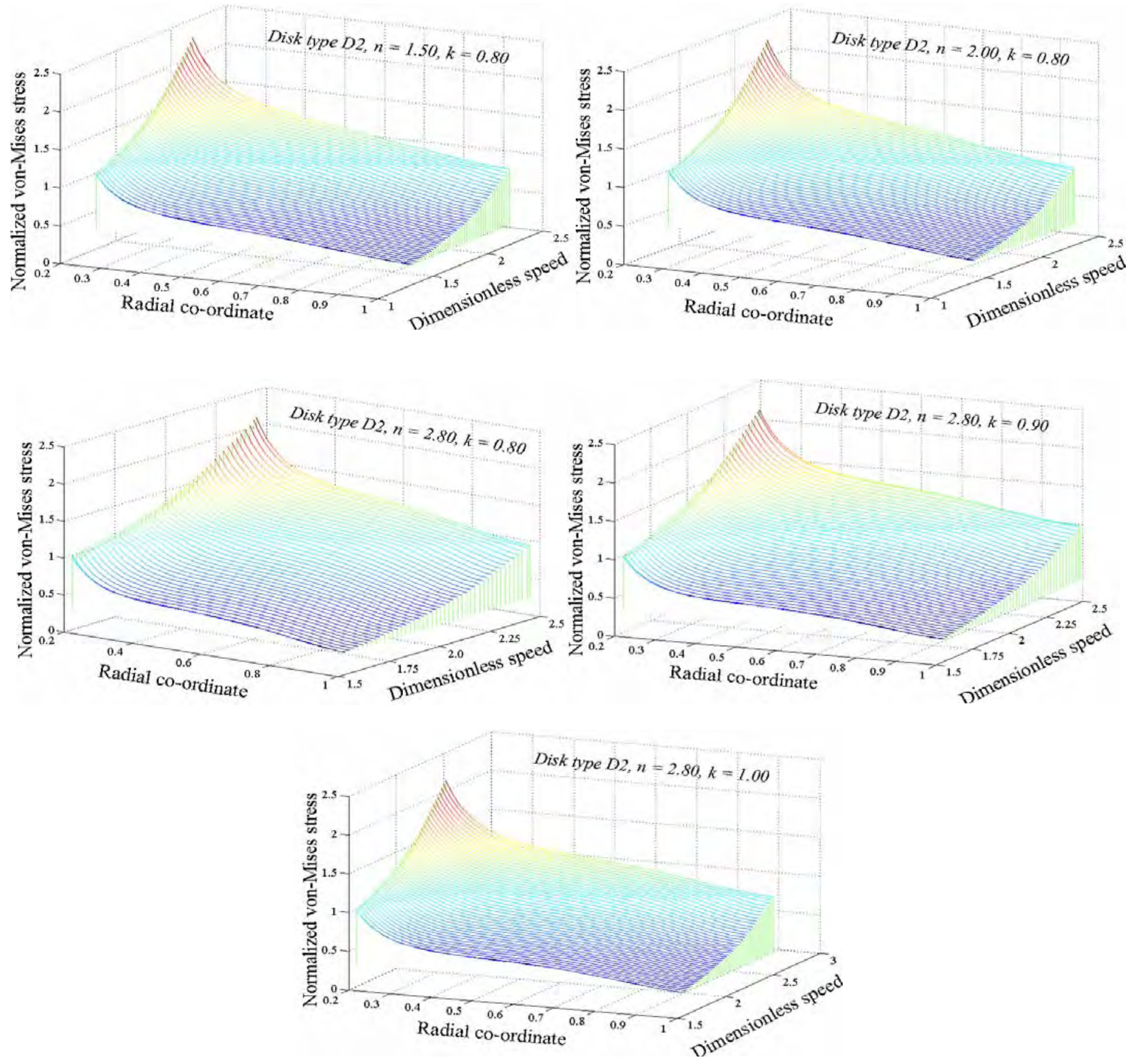

Figure 13a-e Waterfall plots of variation of von-Mises stress with angular speed for various exponential annular disks 

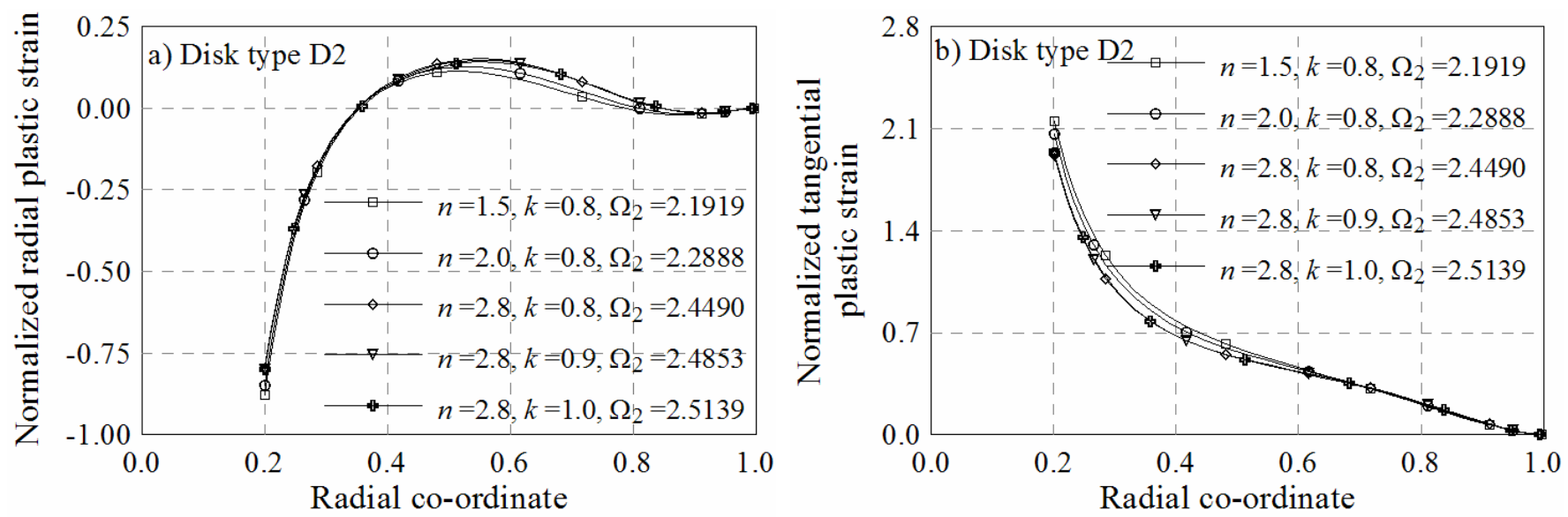

Figure 14a-b Plots of radial and tangential plastic strain for different disk geometries of disk type D2

\section{Nomenclature}

$a$

b

$c_{i}$

$e, p$

$E, E_{1}$

$\{f\}$

$h_{0}, h$

$\bar{H}$

$[K]$

$n, k$

$n f$

$r, t$

$r_{y}$

$u$

$U$

$U_{e}, U_{p}$

V

$\delta$

$\rho$

$v, v_{p}$

$\omega, \Omega$

$\Omega_{1}, \Omega_{2}$

$\varepsilon_{r}, \varepsilon_{t}$

$\varepsilon_{r}^{0}, \varepsilon_{t}^{0}$

$\varepsilon_{r}^{p}, \varepsilon_{t}^{p}$

$\sigma_{r}, \sigma_{t}$

$\sigma_{r}^{0}, \sigma_{t}^{0}$

$\sigma_{r}^{p}, \sigma_{t}^{p}$

$\sigma_{v m}$

$\sigma_{y}$

$\phi_{i}$

$\xi$

$\xi_{1}, \xi_{2}$

$\Delta, \Delta_{1}, \Delta_{2}$

Inner radius of annular disk

Outer radius of the disk

The vector of unknown coefficients

Superscripts, correspond to elastic and plastic state

Elasticity modulus and Tangent modulus of the disk material

Load vector

Thickness at the root and thickness at any radius $r$ of the disk

Normalized hardening parameter

Stiffness matrix

Parameters controlling the thickness variation of disk

Number of functions used to approximate displacement field

Subscripts, correspond to radial and tangential directions

Radial location of yield front

Displacement field of the disk

Strain energy of the disk

Strain energy in the elastic and plastic part of the disk respectively

Work potential of the disk because of rotation

Variational operator

Density of the disk material

Poisson's ratio of the disk material in elastic and post elastic regions

Angular speed and dimensionless angular speed of the disk

Elastic and fully plastic limit angular speed

Strains in radial and tangential direction

Strains in radial and tangential direction in yield condition

Part of radial and tangential strain above yield strain

Radial and tangential stresses

Stresses in radial and tangential direction in yield condition

Part of radial and tangential stress above yield strain

von Mises stress

Yield stress of the disk material

The set of orthogonal functions used to approximate displacement field

Normalized global radial co-ordinate

Normalized local radial co-ordinate in plastic and elastic region

Parameters used to normalize radial coordinate 


\section{Acknowledgement}

The present study is financially supported by CSIR, India (Ref. File/letter No. 9/96[514]2K7-EMR-I) and the support is duly acknowledged by the first author.

\section{References}

Alexandrova N. and Alexandrov S., 2004. Elastic-plastic stress distribution in a rotating annular disk. Mechanics Based Design of Structures and Machines. Taylor and Francis, Vol.32, pp. 1-15.

Alexandrova N., Alexandrov S., and Vila Real P. M. M., 2004. Displacement field and strain distribution in a rotating annular disk. Mechanics Based Design of Structures and Machines. Taylor and Francis, Vol.32, pp. 441-457.

Alexandrova N. and Vila Real P. M. M., 2007. Elastic-plastic stress distributions and limit angular velocities in rotating hyperbolic annular discs. Proceedings of the Institution of Mechanical Engineers, Part C: Journal of Mechanical Engineering Science. Professional Engineering Publishing, Vol.221, pp. 137-142.

Apatay T. and Eraslan A. N., 2003. Elastic deformation of rotating parabolic disks: analytical solutions. Journal of the Faculty of Engineering and Architecture of Gazi University. Gazi University, Vol.18(2), pp. 115-135.

Bhowmick S., Misra D. and Saha K. N., 2008. Approximate solution of limit angular speed for externally loaded solid rotating disk. International Journal of Mechanical Sciences. Elsevier, Vol.50, pp. 163-174.

Bhowmick S., Das D. and Saha K. N., 2006. A study on the elasto-plasticbehavior of a rotating solid disk having variable thickness. Proceedings of International Congress of Computational Mechanics and Simulation. IIT Guwahati, India, pp. 825832.

Chaudhary H. R. and Gupta U.S., 1992. Rotation of hyperelastic annular and solid disks of variable thickness. International Journal of Non-Linear Mechanics. Elsevier, Vol.27(3), pp. 341-346.

Eraslan A. N. and Argeso H., 2002. Limit angular velocities of variable thickness rotating disks. International Journal of Solids and Structures. Elsevier, Vol.39(12), pp. 3109-3130.

Güven U., 1992. Elastic-plastic stresses in a rotating annular disk of variable thickness and variable density. International Journal of Mechanical Sciences. Elsevier, Vol.34, pp. 133-138.

Ma, G., Hao, H., Miyamoto, Y., 2001. Limit angular velocity of rotating disc with unified yield criterion. International Journal of Mechanical Sciences. Elsevier, Vol.43, pp. 1137-1153.

\section{Biographical notes}

Shubhankar Bhowmick received his B.E in Mechanical Engineering from Bhilai Institute of Technology, Durg, in 1999 and ME in Mechanical Engineering (Machine Design) from Jadavpur University, Kolkata, in 2005. He is currently working as Lecturer, Department of Mechanical Engineering, National Institute of Technology, Raipur, Chattisgarh (India). He is engaged in teaching and research activities since the last 9 years. He was awarded with CSIR (SRF) Fellowship in the year 2007. His research area includes Stress and Deformation analysis, Investigation of static and dynamic behavioral analysis of axis-symmetric structures in elastic and post-elastic regime using variational principle. He is also actively engaged in FEA based industrial consultancy projects.

Dipten Misra is a Lecturer in the Department of Mechanical Engineering, Jadavpur University. His principal research area includes design of heat exchangers, heat transfer, finite element analysis and laser materials processing. Presently he is engaged in development of an experimental facility for validation of critical leak rate models and thermal fatigue monitoring system. He is also involved in simulation and experimental validation of laser cutting, welding, forming and laser cleaning of archaeological objects and items of cultural heritage. He has supervised a number of post graduate theses and research projects. He has several publications in international journals and conference proceedings.

Kashi Nath Saha served Indian industry as design engineer in the sectors involving tea machineries, industrial fan and air handling equipment and other heavy machinery components for five years. He joined academia as lecturer in the Department of Mechanical Engineering, Jadavpur University in 1987. He is pursuing teaching and research in the area of machine elements design, stress, deformation analysis and design of structural elements and structural stability analysis. He has also carried out several experimental studies in the field of structural dynamics and supervised a number of post graduate thesis and research projects and has over 50 publications in international journals and conference proceedings.

Received November 2009

Accepted November 2009

Final acceptance in revised form December 2009 